Louisiana State University

LSU Digital Commons

Faculty Publications

Department of Biological Sciences

$1-13-2004$

\title{
Crystal Structure of the Ancient, Fe-S Scaffold IscA Reveals a Novel Protein Fold
}

\author{
Patrick W. Bilder \\ Louisiana State University \\ Huangen Ding \\ Louisiana State University \\ Marcia E. Newcomer \\ Louisiana State University
}

Follow this and additional works at: https://digitalcommons.Isu.edu/biosci_pubs

\section{Recommended Citation}

Bilder, P., Ding, H., \& Newcomer, M. (2004). Crystal Structure of the Ancient, Fe-S Scaffold IscA Reveals a Novel Protein Fold. Biochemistry, 43 (1), 133-139. https://doi.org/10.1021/bi035440s

This Article is brought to you for free and open access by the Department of Biological Sciences at LSU Digital Commons. It has been accepted for inclusion in Faculty Publications by an authorized administrator of LSU Digital Commons. For more information, please contact ir@lsu.edu. 


\title{
Crystal Structure of the Ancient, Fe-S Scaffold IscA Reveals a Novel Protein Fold ${ }^{\dagger}$
}

\author{
Patrick W. Bilder, $₫$ Huangen Ding, and Marcia E. Newcomer* \\ Department of Biological Sciences, Louisiana State University, Baton Rouge, Louisiana 70803 \\ Received August 12, 2003; Revised Manuscript Received October 24, 2003
}

\begin{abstract}
IscA belongs to an ancient family of proteins responsible for iron-sulfur cluster assembly in essential metabolic pathways preserved throughout evolution. We report here the $2.3 \AA$ resolution crystal structure of Escherichia coli IscA, a novel fold in which mixed $\beta$-sheets form a compact $\alpha-\beta$ sandwich domain. In contrast to the highly mobile secondary structural elements within the bacterial $\mathrm{Fe}-\mathrm{S}$ scaffold protein IscU, a protein which is thought to have a similar function, the great majority of the amino acids that are conserved in IscA homologues are located in elements that constitute a well-ordered fold. However, the 10-residue $\mathrm{C}$-terminal tail segment that contains two invariant cysteines critical for the $\mathrm{Fe}-\mathrm{S}$-binding function of a cyanobacterial (Synechocystis PCC) IscA homologue is not ordered in our structure. In addition, the crystal packing reveals a helical assembly that is constructed from two possible tetrameric oligomers of IscA.
\end{abstract}

Iron-sulfur $(\mathrm{Fe}-\mathrm{S})^{1}$ cluster proteins are among the most ancient, highly conserved macromolecules. They function in diverse biochemical processes that include iron homeostasis, electron transfer, metabolic catalysis, nitrogen fixation, regulation of gene expression, and the detection of reactive oxygen species $(1,2)$. This wealth of activities relies upon protein-bound $\mathrm{Fe}-\mathrm{S}$ complexes, which exhibit a wide variation in geometry, oxidation state, and chemical reactivity. Although the physicochemical constitution of biological $\mathrm{Fe}-\mathrm{S}$ clusters has been extensively characterized through synthesis of model structures, spectroscopy, and X-ray crystallography, the mechanisms underlying their biosynthesis are currently in an early phase of exploration. Recent genetic studies have demonstrated an essential nature for gene products of the bacterial iron-sulfur cluster (isc) operon and a corresponding set of predominantly mitochondrial eukaryotic homologues (3-7). Complementary biochemical analyses of these proteins suggest that they function to coordinate the binding and assembly of $\mathrm{Fe}-\mathrm{S}$ clusters that are ultimately transferred to critical $\mathrm{Fe}-\mathrm{S}$ containing target proteins $(8-$ 14). Disruption of both isc genes and their eukaryotic orthologues substantially reduces the activity of the $\mathrm{Fe}-\mathrm{S}$ cluster containing enzymes and oxygen/iron sensor proteins with central roles in the regulation of the citric acid cycle or the electron transport machinery $(3,4,6,7)$. In current models, $\mathrm{Fe}-\mathrm{S}$ cluster biosynthesis in the bacterial cytosol is initiated by IscS, a cysteine desulfurase that generates sulfane sulfur and transfers sulfur to the surface of IscU or IscA, homodimeric scaffold proteins that mediate sequential

\footnotetext{
Supported in part by grants from the NIH (GM55420, M.E.N.), the Louisiana Board of Regents (H.D.), and the Governor's Biotechnology Initiative (M.E.N.).

* To whom correspondence should be addressed. Phone: (225) 5787383. Fax: (225) 578-7258. E-mail: newcomer@lsu.edu.

Department of Biochemistry, Vanderbilt University School of Medicine, Nashville, Tennessee 37235.

${ }^{1}$ Abbreviations; $\mathrm{Fe}-\mathrm{S}$, iron-sulfur; IscA/U/S, iron-sulfur cluster protein $\mathrm{A} / \mathrm{S} / \mathrm{U}$; Fdx, ferredoxin; DTT, dithiothreitol; $\mathrm{HgCl}_{2}$, mercury (II) chloride; PSM, pseudo-symmetric motif.
}

assembly of transiently bound $[2 \mathrm{Fe}-2 \mathrm{~S}]$ and $[4 \mathrm{Fe}-4 \mathrm{~S}]$ clusters (15-17). Holo-IscA/IscU and eukaryotic homologues of IscA/IscU (IsA/IsU) have been shown to complex and transfer $[2 \mathrm{Fe}-2 \mathrm{~S}]$ clusters to the apo-form of a conserved [2Fe-2S] ferredoxin ( $\mathrm{Fdx}$ in Escherichia coli), also synthesized from the isc operon $(12,17-19)$. A recent NMR study of bacterial (Thermotoga maratima) IscU describes a unique metallochaperone structure that, as a result of inherent conformational flexibility, lacks stable tertiary structure in both the unbound and the $\mathrm{Fe}-\mathrm{S}$ cluster-bound states (20). Deuterium exchange measurements coupled with ${ }^{1} \mathrm{H}-{ }^{15} \mathrm{~N}$ HSQC spectra positioned two of three conserved cysteine ligands (C38 and C63) in a proximal arrangement within the $\mathrm{Fe}-\mathrm{S}$ cluster-binding pocket, an area of defined, albeit dynamic, loop regions immediately flanking ordered secondary structural elements.

Three invariant cysteine residues in IscA/IsA have also been implicated in $\mathrm{Fe}-\mathrm{S}$ cluster binding. Mutagenesis of any of these cysteines (present in IscA/IsA proteins as $\mathrm{C} 1-\mathrm{Xn}$ $\mathrm{C} 2-\mathrm{X}_{1}-\mathrm{C} 3$ ) in the yeast mitochondrial IscA homologue, Isa1p, which bears $50 \%$ similarity to IscA, produced similar mitochondrial defects and inhibited growth on nonfermentable carbon sources, consistent with a function for these residues in $\mathrm{Fe}-\mathrm{S}$ cluster ligation (21). In addition, two of the three invariant cysteines ( $\mathrm{C} 2$ and $\mathrm{C} 3$ ) in a cyanobacterial IscA (the Synechocystis PCC 6803 slr1417 gene product), a protein that purifies from this organism as an iron-bound species, were shown to be essential for the coordination of a $[2 \mathrm{Fe}-2 \mathrm{~S}]$ cluster, whereas alanine substitution of $\mathrm{C} 1$ weakened cluster formation by $40 \%(17,22)$. The absorption and Mossbauer spectra that supported these findings were consistent with tetrahedral thiolate ligation of one $[2 \mathrm{Fe}-$ $2 \mathrm{~S}$ ] cluster per dimer by $\mathrm{C} 2$ and $\mathrm{C} 3$ or one $\mathrm{Fe}-\mathrm{S}$ cluster bound between monomers. Similarly, serine substitution of C1, C2, or C3 in Schizosaccharomyces pombe IsA significantly weakened $[2 \mathrm{Fe}-2 \mathrm{~S}]$ cluster binding (23).

Our laboratory has recently solved and refined the crystal structure of $E$. coli IscA, a $12.8 \mathrm{kDa}, 107$-residue polypeptide 
Table 1: Data Collection and Phasing Statistics

\begin{tabular}{|c|c|c|c|c|c|c|}
\hline dataset & $\begin{array}{l}\text { resolution } \\
(\AA)\end{array}$ & $\begin{array}{c}\text { completeness } \\
(\%)\end{array}$ & $\begin{array}{c}\text { Diffraction Data Statistics }{ }^{a} \\
\text { total/unique } \\
\text { reflections }\end{array}$ & $I / \sigma$ & redundancy & $\begin{array}{c}R_{\text {sym }}{ }^{b} \\
(\%)\end{array}$ \\
\hline native & $\infty-2.65$ & $99.8(100.0)$ & $77,724 / 8,034$ & $58(5.5)$ & $9.7(9.9)$ & $6.6(47.9)$ \\
\hline $\mathrm{HgCl}_{2}$ & $\infty-2.30$ & $99.4(99.1)$ & $67,990 / 12,243$ & $38(3.7)$ & $5.6(5.3)$ & $6.3(45.7)$ \\
\hline
\end{tabular}

\begin{tabular}{|c|c|c|c|c|c|c|c|c|c|}
\hline \multirow[b]{3}{*}{ derivative } & \multirow{3}{*}{$\begin{array}{l}\text { binding } \\
\text { sites }\end{array}$} & \multirow{3}{*}{$\begin{array}{l}\text { occupancy } \\
\text { (site } 1 / 2 / 3 \text { ) }\end{array}$} & \multicolumn{3}{|c|}{ Phasing Statistics- SIR } & \multirow{2}{*}{\multicolumn{2}{|c|}{ Rcullis $^{d}$}} & \multirow{2}{*}{\multicolumn{2}{|c|}{$\mathrm{FOM}^{e}$}} \\
\hline & & & \multirow{2}{*}{$\begin{array}{l}\text { resolution } \\
(\AA)\end{array}$} & \multicolumn{2}{|c|}{ phasing power ${ }^{c}$} & & & & \\
\hline & & & & acentric & centric & \multicolumn{2}{|c|}{ ntric $\quad$ cer } & acentric & centric \\
\hline $\mathrm{HgCl}_{2}$ & 3 & $0.89 / 0.88 / 0.11$ & $27-2.8$ & 1.54 & 1.27 & 0.66 & 0.67 & 0.36 & 0.63 \\
\hline
\end{tabular}

${ }^{a}$ The values in parentheses are for the highest resolution shell. ${ }^{b} R_{\mathrm{sym}}=\sum(I-\langle I\rangle) / \sum(I)$, where $I$ is the intensity measurement for a given reflection and $\langle I\rangle$ is the average intensity for multiple measurements of this reflection. ${ }^{c}$ Phasing power equals heavy atom structure factor divided by the root-mean-square lack of closure error (statistics from CNS). ${ }^{d}$ Rcullis equals the lack of closure error divided by the iso-ano difference (generalized Rcullis in CNS). ${ }^{e} \mathrm{FOM}$ is the figure of merit.

with a novel fold, to $2.3 \AA$ resolution. A C-terminal decapeptide that contains two of the three invariant cysteines (C99 and C101) required for $\mathrm{Fe}-\mathrm{S}$ binding in the Synechocystis PCC 6803 slr1417 protein is not visible in the electron density map. The lack of well-defined electron density for this region is consistent with either a dynamic disorder or multiple ordered conformations of the region. A comparison of the IscA structure with the $\mathrm{Fe}-\mathrm{S}$ scaffold IscU is provided.

\section{MATERIALS AND METHODS}

Crystallization and Data Collection. E. coli IscA was overexpressed from a C-terminal, 6-His-tagged construct and purified to homogeneity as performed by Ding and coworkers (Ding et al., manuscript submitted). IscA was subsequently desalted using a PD10 column, concentrated to $17 \mathrm{mg} / \mathrm{mL}$, and incubated at $4{ }^{\circ} \mathrm{C}$ overnight with $1.1 \mathrm{mM}$ ferrous ammonium sulfate and $2 \mathrm{mM}$ DTT. Under a glovebag enclosing a helium atmosphere, crystals were grown at room temperature by the vapor diffusion method in drops containing $0.8-1.0 \mathrm{M}$ dibasic ammonium phosphate buffered with $0.1 \mathrm{M}$ imidazole, $\mathrm{pH}$ 8.0. Hexagonal, rod-shaped crystals of the dimensions $\sim 0.2 \times 0.2 \times 0.7 \mathrm{~mm}$ appeared in the presence of $10 \mathrm{mM}$ mercury (II) chloride $\left(\mathrm{HgCl}_{2}\right)$ supplemented in the mother liquor (derivative conditions) or its absence (native conditions) and grew to near maximal size within 2-4 weeks. IscA crystals belong to the space group $P 6_{2}$ with unit cell lengths $a=55.5$ and $c=159.0 \AA$. The estimated solvent content is $55 \%$ with a dimer in the asymmetric unit.

Diffraction data for a single native and derivative crystal were collected at $100 \mathrm{~K}$ on a Mar345 image plate detector mounted on a Nonius FR591 rotating $\mathrm{CuK} \alpha$ anode fitted with Osmic mirrors. Crystals were transferred to a cryoprotectant solution containing $20 \%$ ethylene glycol/ $/ 80 \%$ mother liquor immediately prior to freezing. The crystal-to-detector distance was $160 \mathrm{~mm}$ for $1^{\circ}$ oscillation images, each exposed for $30 \mathrm{~min}$. Final statistics from intensity data processed with Denzo and scaled in Scalepack are listed in Table 1 (24). The high redundancy and signal-to-noise ratio of the diffraction data allowed us to include reflections to resolutions of $2.65 \AA$ (native) and $2.3 \AA$ (derivative) in the final refinements.

Structure Determination and Refinement. Cocrystallization of IscA with $\mathrm{HgCl}_{2}$ noticeably enhanced the size, mor- phology, and diffraction quality of the crystals. An overall $R_{\text {merge }}$ of $18.0 \%$ for native and $\mathrm{HgCl}_{2}$-IscA structure factor amplitudes to $2.7 \AA$ was indicative of a heavily substituted derivative. Three heavy atom-binding sites in the asymmetric unit, two of which appeared definitively on the Harker sections and refined to high occupancy values, were identified through an automated Patterson search in CNS (25) and used to calculate initial SIR phases to $2.8 \AA$ (Table 1). An electron density map calculated from solvent-flattened SIR phases (using the program DM in CCP4 (26), overall Figure of Merit $=0.47$ ) revealed, after skeletonization in MAPMAN (27) and visualization in $\mathrm{O}(28)$, salient secondary structural features. One pair of overlapping, albeit disjointed, $\beta$-sheets was sufficient to elucidate a 2 -fold noncrystallographic symmetry (NCS) axis and to generate an initial matrix for the production of an improved, averaged map in DM. This map served as a starting point for the construction of a polyalanine backbone. After iterative cycles of model building in $\mathrm{O}$, SigmaA weighting, phase combination, solvent flattening, phase extension in $\mathrm{CCP} 4$, and rigid body refinement and energy minimization in CNS, a majority of the side chains were identified.

In the early stages of refinement and interpretation of $2 F_{\mathrm{o}}-F_{\mathrm{c}}$ maps, the structure factor amplitude array from the higher resolution derivative dataset was employed. As $R$ factors dropped below 0.30 , it became clear that independent monomers were nearly identical, and the model improved as high NCS restraint weights (400 in CNS) were imposed. Further refinement cycles that included energy minimization, $B$ group refinement, and simulated annealing were conducted using maximum likelihood refinement in CNS. Concluding stages of refinement were carried out in REFMAC5 (CCP4) (29). Monomers in the final models lack the last 10 residues present in the native protein, including Cys99 and Cys101. A Ramachandran plot in PROCHECK (30) demonstrates favorable main-chain geometry for both the native and the $\mathrm{Hg}$-IscA structures with the exception of a single nonglycine outlier, Cys35, in the native model. In a $2 F_{\mathrm{o}}-F_{\mathrm{c}}$ omit map produced from the derivative dataset, density corresponding to each Cys35 thiol is enveloped in a $17 \sigma \mathrm{Hg}$ peak, consistent with covalent binding of the metal at this position. The final $R / R_{\text {free }}$ for the native and derivatized structures are 23.2/25.6 and $22.9 / 26.3 \%$. Refinement statistics are summarized in Table 2. 


\begin{tabular}{lll}
\hline Table 2: Refinement Statistics & & \\
\hline & native & $\mathrm{HgCl}_{2}$ \\
\hline resolution $(\AA)$ & $27-2.6$ & $27-2.3$ \\
no. of reflections & 7371 & 11110 \\
$\sigma$ cutoff & none & none \\
$R_{\text {factor }}(\%)$ & 23.2 & 22.9 \\
$R_{\text {free }}(\%)^{\mathrm{a}}$ & 25.6 & 26.3 \\
no. of refined atoms & & \\
$\quad$ protein & 1470 & 1470 \\
$\quad$ water & 61 & 85 \\
average $B$ factors $\left(\AA^{2}\right)$ & & \\
$\quad$ protein & 42.0 & 43.7 \\
$\quad$ water & 40.2 & 43.6 \\
$\quad$ Hg & $\mathrm{NA}$ & 72.2 \\
$\quad$ overall & 42.0 & 43.8 \\
$B$ from Wilson plot & 56.6 & 44.2 \\
$\quad$ rms deviations & & \\
$\quad$ bonds $(\AA)$ & 0.010 & 0.014 \\
$\quad$ angles $($ deg $)$ & 1.4 & 1.5 \\
Ramachandran plot & & \\
$\quad$ most favored regions $(\%)$ & 93.8 & 94.4 \\
$\quad$ additional allowed $(\%)$ & 4.9 & 5.6 \\
$\quad$ disallowed $(\%)$ & $1.2^{b}$ & 0.0 \\
\hline
\end{tabular}

${ }^{a} R_{\text {free }}$ was calculated from $5 \%$ of the diffraction data and monitored throughout refinement. ${ }^{b}$ Corresponds to residue cysteine 35 from both monomers in the asymmetric unit.

\section{RESULTS AND DISCUSSION}

Overall Fold. The absence of proteins in the Dali database that bear significant structural homology to IscA suggests that IscA adopts a novel protein fold. This fold is characterized by a striking, internal 2-fold symmetry such that the unique portion of the structure covers roughly $50 \%$ of the primary sequence. A complete IscA monomer (Figure 1A) includes tandem pseudo-symmetric motifs (PSM) 1 ( $\beta 1-\alpha 1$ $\beta 2-\beta 3)$ and $2(\beta 5-\alpha 2-\beta 6-\beta 7)$ separated by a unique, nearpalindromic, 14 amino acid stretch ( E $_{43}$ FVDEPTPEDIVFE $\left._{56}\right)$ encompassing the three $\mathrm{C}$-terminal residues of $\beta 3$, a short, 6-residue loop region, and five of the six residues that constitute $\beta 4$ (Figure 1C). PSM1 and PSM2 each resemble a fish hook twisted at the bend so as to create a $45^{\circ}$ angle between the barb and the shank (Figure 1B). In PSM 1/2, $\beta 1 / \beta 5$ lies along the barb, $\alpha 1 / \alpha 2$ traverses the bend, and $\beta 2$ $\beta 3 / \beta 6-\beta 7$ extend the shank as a twisted, antiparallel $\beta$-sheet. If the Isc A monomer constitutes the fundamental building block of a ladder that represents an oligomeric state (Figure 2A,B), the hooks are interlocked such that the barb of PSM1 $(\beta 1)$ points inward at a $45^{\circ}$ angle, the bend of PSM1 $(\alpha 1)$ rests on the side rail, and its shank ( $\beta 2$ and $\beta 3$ ) stretches the length of the upper rung. The barb of PSM2 ( $\beta 5)$ lies atop the shank of PAM1 ( $\beta 2)$ from its midpoint to the side rail, its bend $(\alpha 2)$ becomes the lower right helix of a circular cavity, and its shank ( $\beta 6$ and $\beta 7$ ) juts downward to fill the distance between joints at an angle perpendicular to the shank of PSM1. The resulting architecture is a $\beta$-sandwich domain composed of two mixed $\beta$-sheets: a 4 -stranded, twisted $\beta 4$ $\beta 5-\beta 2-\beta 3$ sheet and a 3 -stranded, twisted $\beta 1-\beta 6-\beta 7$ sheet, offset by $50^{\circ}$ to achieve tight extensive hydrophobic packing at the core.

Examination of the crystal packing of IscA monomers reveals a super-helical assembly with a radius of $25 \AA$ and a pitch delimited by three arbitrarily designated, tetrameric assemblies, each translated $50 \AA$ along the helical axis (Figure 2B). Upon this axis, the IscA monomer follows a
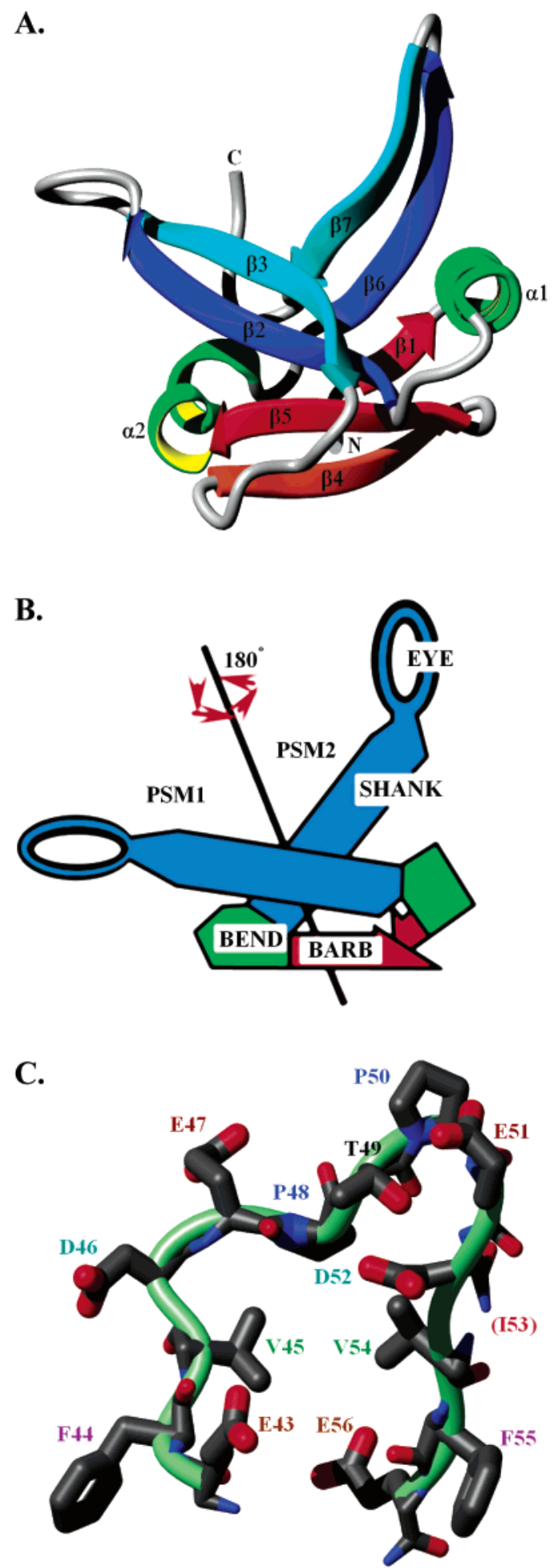

FIGURE 1: Unique pseudo-symmetric fold of IscA. (A) A ribbon diagram showing the secondary structural elements in IscA. (B) A schematic representation of the monomer fold as a pair of interlocked fish hooks. Each hook represents a single pseudosymmetric motif (PSM). Elements related by pseudo-2-fold symmetry are colored identically in panels $\mathrm{A}$ and $\mathrm{B}(\beta 1, \beta 5$, and barbsred; $\alpha 1, \alpha 2$, and bend-green; $\beta 2$ and $\beta 6-$ blue; $\beta 3$ and $\beta 7-$ cyan; and shank-light blue). The $\beta-4$ strand, which is cut by the pseudo2 -fold axis, is depicted in brown. (C) Structure of the 14-residue pseudo-palindromic amino acid sequence. Identifiers for residues related on the virtual 2-fold are colored identically (F44/55-violet, E43/56-brown, V45/54-green, D46/52-turquoise, E47/51-brick red, and P48/50-blue). T49, at the palindrome center, is black. Residue I53 (labeled red in parentheses) is not shown. Panels A and C were drawn using Molmol (34) and rendered in Povray (35). 
A.

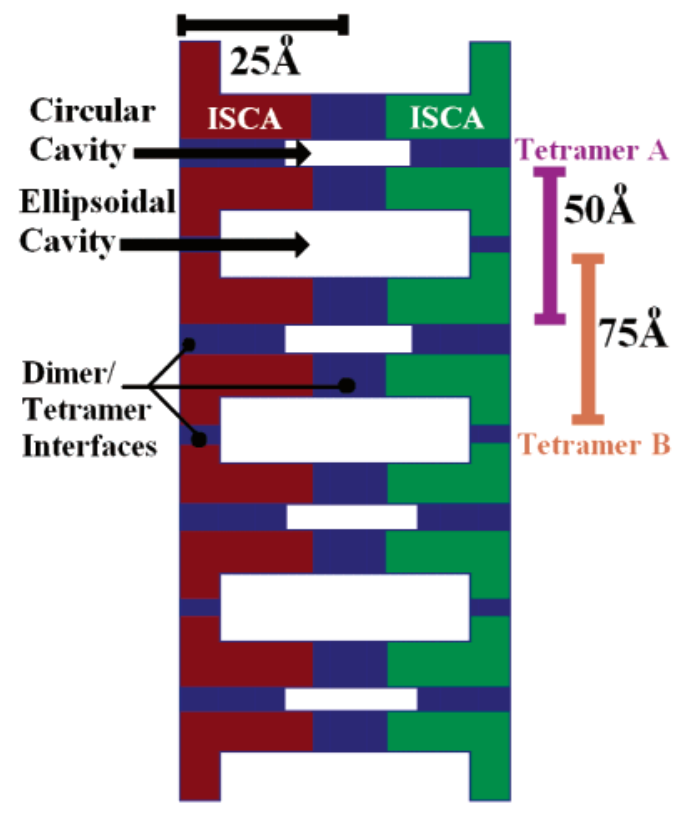

B.

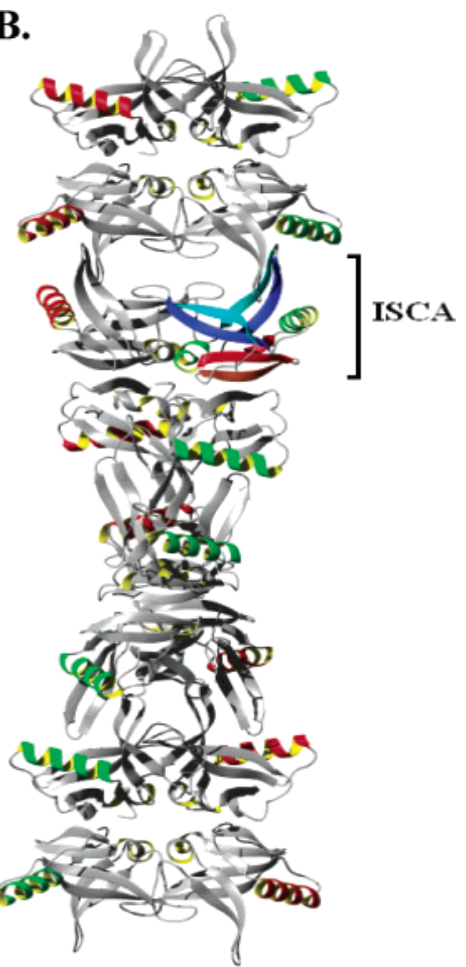

C.
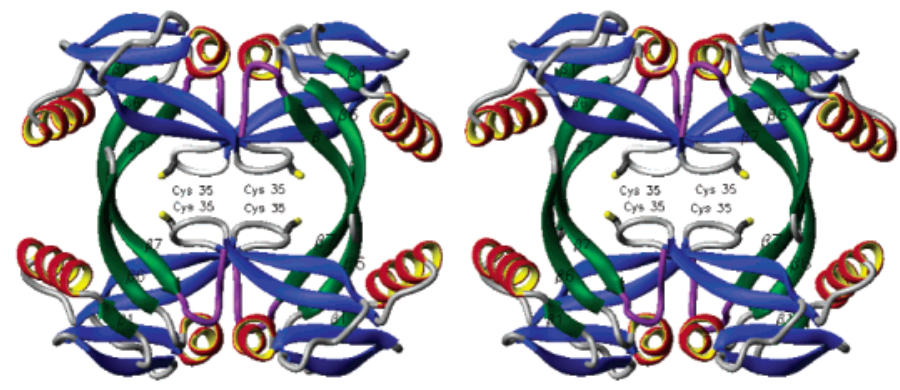

D.
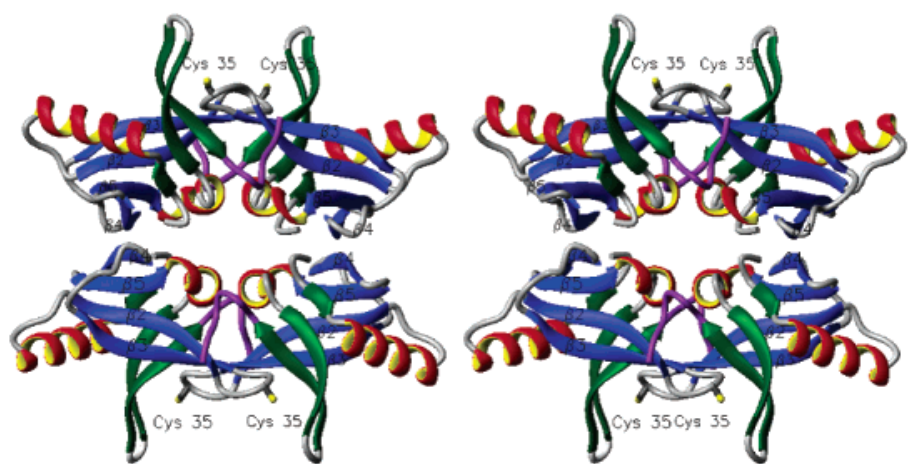

FIGURE 2: Structure of IscA oligomers. Cartoon representation (A) of the IscA super-helix (B) as a ladder. Brackets designate the distance along the pitch or diameter of the IscA ladder. In panel B, the IscA monomer is colored using the scheme from Figure 1A to highlight its position and orientation in the superstructure. (C) Tetramer A. The 6-stranded, mixed $\beta$-sheet unique to tetramer A is labeled and shown in green. (D) Tetramer B. The 16-stranded $\beta$-barrel unique to tetramer B is labeled and shown in blue. Panels $\mathrm{A}$ and $\mathrm{C}$ were drawn using Molmol (34) and rendered in Povray (35).

spiraling, ladder-like pathway stabilized by dimer/tetramer interfaces that occur in regions resembling either joints or the midpoints of adjacent rungs or interceding side rails of the ladder. Two distinct tetrameric arrangements are possible: one in which four monomers surround a central ellipsoidal cavity $\sim 45 \AA$ wide and $7 \AA$ tall (for the sake of discussion, tetramer $\mathrm{A}$ ) and one in which the monomers surround a cylindrical cavity with a $5 \AA$ radius (tetramer B). Both arrangements are dimers of dimers. In tetramer A, the monomers are positioned at the four corners of a picture frame, and each contributes the shanks of the fish hooks to its neighbors (Figure 2C). PSM 1 forms a semicircular, 8 -stranded $\beta$-sheet and PSM 2 forms a 4 -stranded $\beta$-sheet with the dimer mates. In tetramer $\mathrm{B}, 4$-stranded $\beta 3-\beta 2-\beta 5$ $\beta 4$ sheets of neighboring monomers are joined through $\beta 4$ $\beta 4$ and $\beta 3-\beta 3$ hydrogen bonds to form $90^{\circ}$ arcs over a topologically circular palindrome that spirals into a 16stranded $\beta$-barrel enclosing the circular cavity (Figure 2D).

Both the purified bacterial protein and its eukaryotic homologues have been shown to exist in dimeric, tetrameric, 

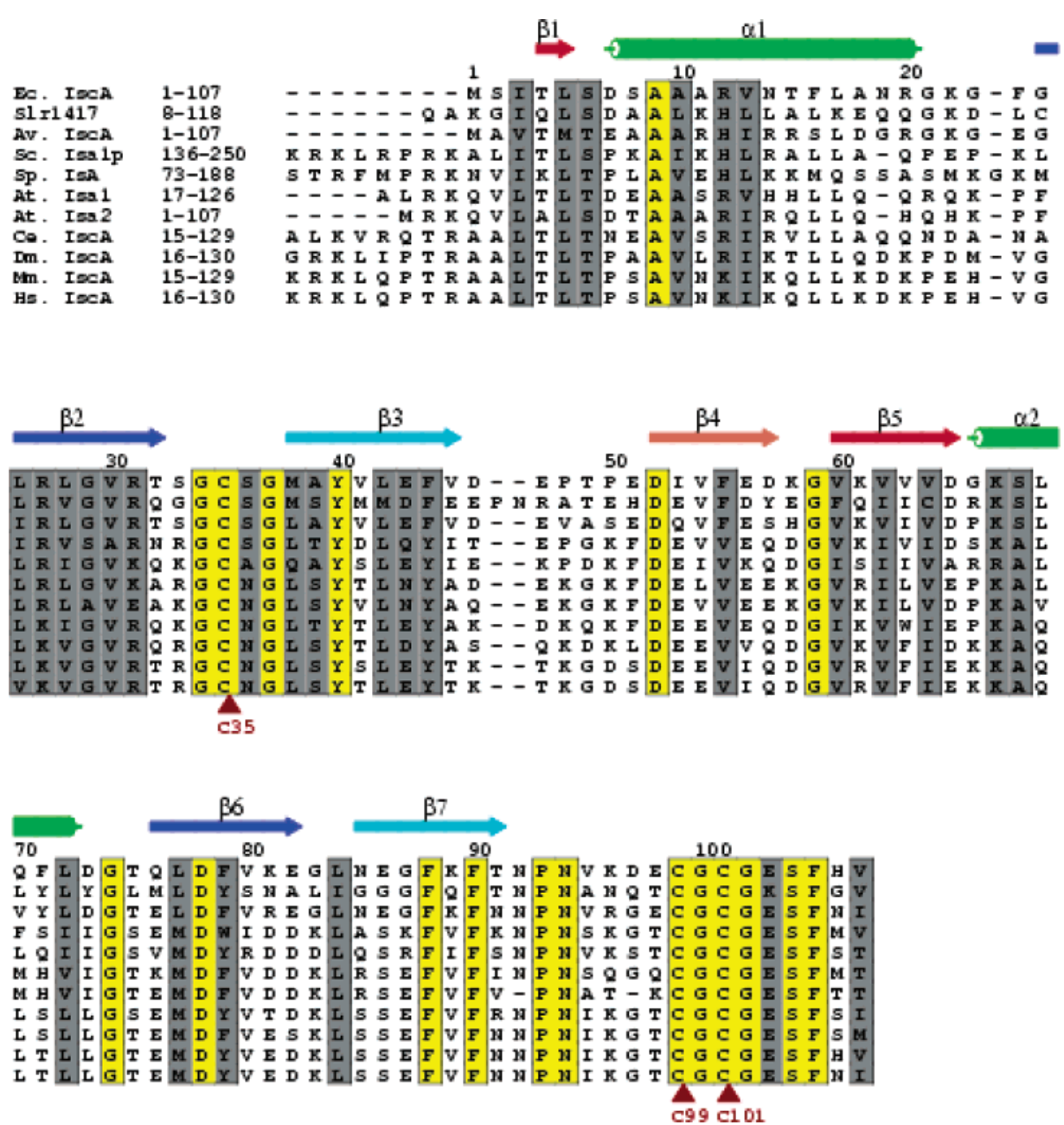

FIGURE 3: Structure-based sequence alignment of IscA orthologues. IscA/IsA from Escherichia coli (Ec), Synechocystis PCC 6803 slr1417 (slr1417), Azotobacter vinelandii (Av), Saccharomyces cerevisiae (Sc), Saccharomyces pombe (Sp), Arabidopsis thaliana (At), Caenorhabditis elegans (Ce), Drosophila melanogaster (Dm), Mus musculus (Mm), and Homo sapiens (Hs). Residues conserved in all sequences are highlighted in yellow, and highly conserved residues are highlighted in gray. Invariant cysteines 35, 99, and 101 of E. coli are marked by brown triangles. Secondary structure elements are indicated above the sequence for $E$. coli IscA and shaded using the same color scheme as Figure 1A. This figure was created using Alscript (36).

or higher order aggregation states $(19,23,31)$, and tetramers A or B represent two possible models for the oligomeric states observed in solution. Each of two tetrameric configurations buries $\sim 25 \%\left(5600-6100 \AA^{2}\right)$ of solvent-accessible surface area in dimer/tetramer interfaces between adjacent IscA subunits, suggesting that either tetramer A or tetramer $\mathrm{B}$ can exist as a physiologically stable entity. The unique areas of contact for the independent assemblies $\left(2000 \AA^{2}\right.$ at the midpoints of side rails composing tetramer A or 1500 $\AA^{2}$ at the mid-rung region that joins dimers from tetramer B) are each sufficient for a functional oligomeric interface. The $\beta 4-\beta 4$ dimerization interface unique to tetramer $\mathrm{B}$ is predominantly stabilized by main-chain hydrogen bonds from I53, V54, and F55 and van der Waals interactions between I53 side chains on opposing strands. The additional surface area that selectively stabilizes tetramer A is characterized by favorable ionic interactions involving the guanidinium group of R12, which extends from the $\alpha-1$ helices of one dimer partner to the mid-side rail E86 carboxylate on $\beta 7$, an edge on face stacking arrangement of F79 and F88 that restrains the linear conformation of the $\beta 6-\beta 7$ shanks as they exit the protein core and the insertion of L84, situated at the eye of the $\beta 6-\beta 7$ shanks, into a dense hydrophobic pocket of F16, L42, and F44 on the opposite monomer. This constellation of residues, and particularly those that are both hydrophobic and highly conserved or invariant (L42, F44, F79, and F88-see Figure 3), maintains the backbones of the $\beta 7$ strands at the borders of a channel enclosing six ordered water molecules. Because of the favorable packing at this interface (a feature that relies upon the specific R12E86 ionic interaction, the conserved hydrophobic network, and the presence of an extensively hydrogen-bonded water channel), we propose that tetramer $\mathrm{A}$ and the dimeric substructure maintaining these contacts represent the most likely oligomeric states in the aqueous milieu of the cell.

Conservation in Sequence and Structure. IscA orthologues maintain a high degree of evolutionary conservation as highlighted by a sequence alignment for representative species from each kingdom (Figure 3). Over the 107 amino acid stretch present in the bacterial forms, $47 \%$ (50) of residues are highly conserved, and 18\% (19) are identities. Most of the highly conserved residues (60\%) are located within secondary structural elements, and a majority of these $(67 \%)$ contribute to the hydrophobic core that seals the twisted $\beta$-sandwich domain of IscA. When viewed from a perspective down the intramolecular 2-fold, the hydrophobic side chains fill a rectangular slab bounded by the $\alpha$-helices on the short edges, the $\beta$-sheets on the long edges, and the 


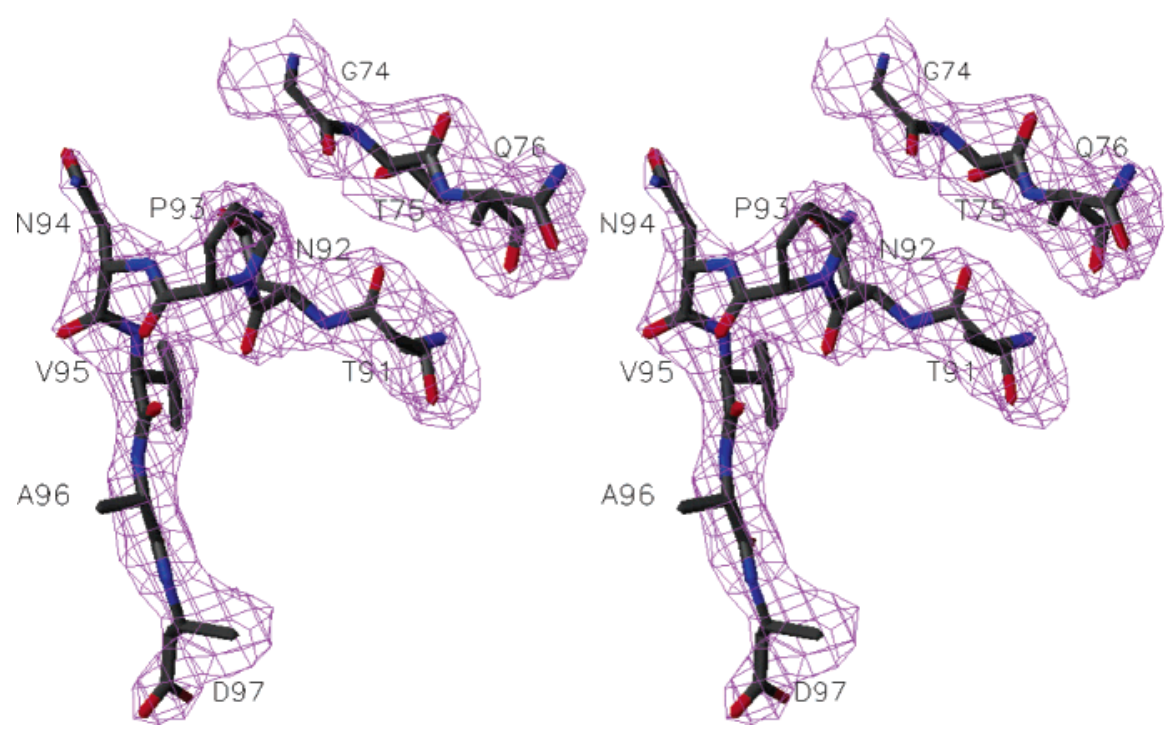

FIGURE 4: Electron density for residues preceding the disordered C-terminus of IscA. Electron density (violet) from final, native $2.65 \AA$ $2 F_{\mathrm{o}}-F_{\mathrm{c}}$ maps contoured at $1 \sigma$ in the region encompassing the last visible C-terminal residues (T91-D97). Residues G74-D76 and hydrogenbonding partners T91-N92 (from the $\beta 6$ - $\beta 7$ shank) lie in a region of well-defined density. Beyond the sharp turn at P93 (N94-D97), sidechain density becomes significantly more diffuse or disappears entirely. Because of the absence of side-chain density for K96, alanine was substituted at this position in this final model. The figure was drawn using Molmol (34) and rendered in Povray (35).

$\beta 4$-strand at its base. Hydrophobic packing of conserved residues F55, I3, L5, A9/10, V60/62/64, L77, L26, L28, V13, L72, F79, L17, F16, F88, F90, F71, F44, L42, Y40, M38, and V30 occurs progressively upward in a knobs in holes type fashion similar to that described for $\alpha$-helices. Accompanying side chains stack tightly near the $\alpha-\beta$ corners or between three and four hydrophobic functional groups protruding from two opposite strands above and below the plane of the knob. Other conserved residues that maintain the integrity of the IscA structure include conserved residues E56( $\beta 4)$ and $\mathrm{S} 6(\beta 1)$ (consensus $\mathrm{S} / \mathrm{T})$, invariant residues D52$(\beta 4)$ and D78( $\beta 6)$, and R27 $(\beta 2) / \mathrm{K} 61(\beta 5)$ (consensus $\mathrm{R} / \mathrm{K}$ ). Intramolecular salt bridges consisting of D52-R27 and E56-K61 tether the conformation of the $\beta 4-\beta 5-\beta 2-\beta 3$ sheet, and a D78-S6 hydrogen bonding interaction secures $\beta 1-\beta 6$ contacts at the turn into the $\alpha 1$ helix. Three invariant glycine residues $\mathrm{G} 37$, G59, and G74 are ostensibly required for transitions into $\beta 3, \beta 5$, and $\beta 6$ as they are located in turns immediately before the initiation of these strands. The placement of conserved residues at critical positions in the tertiary structure suggests that the overall fold of IscA has been strictly preserved throughout evolution.

Cysteine Pocket. Only the first of the three invariant cysteines (C35, C99, and C101) implicated in the ligation of iron-sulfur cluster complexes is visible in the electron density. C35 is located within two bulging pockets (10 tall) found on each side of the centrally compressed ( $7 \AA$ tall) ellipsoidal cavity of tetramer A (Figure 2C). On both faces of the cavity, C35 projects from monomers on diagonally opposite corners of the frame (related by the dimer 2-fold) into opposite bulges from a flexible, conserved GCXG loop between $\beta 2$ and $\beta 3$. At one face, $\mathrm{C} 35$ residues are separated by $20 \AA$ and located at equivalent positions, each at the eye of a PSM2 shank protruding from the cavity roof and floor. In the native structure, the $B$ factor for the $\mathrm{GC}_{35} \mathrm{XG}$ region deviates substantially from the mean protein $B$ factor (+1.6-fold), and C35 is a unique Ramachandran outlier. In contrast, this same region is well-ordered in the $\mathrm{Hg}$-derivatized structure (the $B$ factor is only 1.2 times greater than the mean protein $B$ factor). Apparently, the covalent binding of $\mathrm{Hg}$ to the labile cysteine imparts rigidity to this flexible loop. In addition, the side chain of E82, located on the $\beta 6$ strand of an adjacent dimer partner, is positioned in the mercury-binding pocket of the derivative and rotated $180^{\circ}$ away from a solvent-exposed orientation observed in the native structure. This motion adjusts the side chain carboxyl from $9 \AA$ (native) to 4 and $3.7 \AA$ from the C35 thiol and mercury, respectively.

A consensus C-terminal stretch CGCGESF containing the other putative iron-binding cysteine residues, C99 and C101, is completely disordered, as judged from the fact that it is not visible in the electron density map. This sequence is located at the end of a coil that begins at the end of $\beta 7$, turns sharply at an invariant proline (P93), and extends superficially down the center of the upper dimer to the last visible residue, D97, at the brink of the central cavity (Figure 4).

Comparison of $\mathrm{Fe}-\mathrm{S}$ Scaffolds IscA and IscU. Although in vitro kinetic and steady-state spectroscopic data suggest that IscU/IsU and IscA/IsA assemble solvent-accessible, labile $\mathrm{Fe}-\mathrm{S}$ clusters and transfer these clusters to apoferredoxin at similar rates, there are noteworthy differences in primary sequence, function, and fold. For example, IscA/ Is A and IscU/IsU are each highly conserved yet share no significant sequence homology, Is A binds $\mathrm{Fe}-\mathrm{S}$ clusters with a higher affinity than IsU, and IsA recognizes a binding site on the target ferredoxin distinct from that recognized by IsU (32). Whereas the high degree of conformational flexibility observed for apo/holo-IscU has been attributed to a series of highly mobile hydrophobic side chains (20), a rigid and extensive hydrophobic network imparts stability to the core of monomeric IscA. In contrast to the global fluidity of IscU, only a few residues within the cysteine pocket or the C-terminal tail were significantly disordered in native IscA. Beyond the dynamic features that distinguish these structures, Isc $\mathrm{A}$ and IscU diverge in both secondary structural content and, insofar as a three-dimensional representation of IscU could be obtained, the organization of these secondary 
structural elements. However, the disorder present in the structures of both IscA and IscU are consistent with a role for these proteins in the assembly and transfer of transient $\mathrm{Fe}-\mathrm{S}$ clusters to various $\mathrm{Fe}-\mathrm{S}$ target proteins $(8,17,19)$. This hypothesis is supported by the observation that all of the three conserved cysteines are located in mobile loop regions of IscA and IscU, at least one of the predicted $\mathrm{Fe}-\mathrm{S}$ cysteine ligands could not be visualized in either structure, and the remaining cysteines were impossible to position precisely within the putative $\mathrm{Fe}-\mathrm{S}$-binding regions of native IscA or apo/holo IscU. In addition, neither IscA nor IscU possess the degree of rigidity characteristic of $\mathrm{Fe}-\mathrm{S}$ cluster or metal-binding sites observed for the majority of known metallochaperone or metalloenzyme structures, many of which bear the common ferredoxin-like or Rossman-type folds $(20,33)$. These $\mathrm{Fe}-\mathrm{S}$ complexes, which function primarily as redox-labile cofactor centers for catalysts or oxygen sensors, are structurally versatile, and with few exceptions, capable of rearrangement with minimal, highly localized conformational changes in the protein ligands that maintain the $\mathrm{Fe}-\mathrm{S}$ cluster (33).

\section{REFERENCES}

1. Beinert, H. (2000) J. Biol. Inorg. Chem. 5, 2-15.

2. Beinert, H., Holm, R. H., and Munck, E. (1997) Science 277, 6539.

3. Schwartz, C. J., Djaman, O., Imlay, J. A., and Kiley, P. J. (2000) Proc. Natl. Acad. Sci. U.S.A. 97, 9009-14.

4. Tokumoto, U., and Takahashi, Y. (2001) J. Biochem. (Tokyo) 130, $63-71$.

5. Lange, H., Kaut, A., Kispal, G., and Lill, R. (2000) Proc. Natl. Acad. Sci. U.S.A. 97, 1050-5.

6. Strain, J., Lorenz, C. R., Bode, J., Garland, S., Smolen, G. A., Ta, D. T., Vickery, L. E., and Culotta, V. C. (1998) J. Biol. Chem. $273,31138-44$.

7. Schilke, B., Voisine, C., Beinert, H., and Craig, E. (1999) Proc. Natl. Acad. Sci. U.S.A. 96, 10206-11.

8. Mansy, S. S., Wu, G., Surerus, K. K., and Cowan, J. A. (2002) J. Biol. Chem. 277, 21397-404.

9. Tokumoto, U., Nomura, S., Minami, Y., Mihara, H., Kato, S., Kurihara, T., Esaki, N., Kanazawa, H., Matsubara, H., and Takahashi, Y. (2002) J. Biochem. (Tokyo) 131, 713-9.

10. Kato, S., Mihara, H., Kurihara, T., Takahashi, Y., Tokumoto, U., Yoshimura, T., and Esaki, N. (2002) Proc. Natl. Acad. Sci. U.S.A. 99, 5948-52.

11. Agar, J. N., Krebs, C., Frazzon, J., Huynh, B. H., Dean, D. R., and Johnson, M. K. (2000) Biochemistry 39, 7856-62.
12. Wu, S. P., Wu, G., Surerus, K. K., and Cowan, J. A. (2002) Biochemistry 41, 8876-85.

13. Yoon, T., and Cowan, J. A. (2003) J. Am. Chem. Soc. 125, 607884.

14. Muhlenhoff, U., Richhardt, N., Gerber, J., and Lill, R. (2002) J. Biol. Chem. 277, 29810-6.

15. Agar, J. N., Zheng, L., Cash, V. L., Dean, D. R., and Johnson, M. K. (2000) J. Am. Chem. Soc. 122, 2136-7.

16. Krebs, C., Agar, J. N., Smith, A. D., Frazzon, J., Dean, D. R., Huynh, B. H., and Johnson, M. K. (2001) Biochemistry 40, 14069-80.

17. Wollenberg, M., Berndt, C., Bill, E., Schwenn, J. D., and Seidler, A. (2003) Eur. J. Biochem. 270, 1662-71.

18. Wu, G., Mansy, S. S., Wu, S. P., Surerus, K. K., Foster, M. W., and Cowan, J. A. (2002) Biochemistry 41, 5024-32.

19. Ollagnier-de-Choudens, S., Mattioli, T., Takahashi, Y., and Fontecave, M. (2001) J. Biol. Chem. 276, 22604-7.

20. Bertini, I., Cowan, J. A., Del Bianco, C., Luchinat, C., and Mansy, S. S. (2003) J. Mol. Biol. 331, 907-24.

21. Kaut, A., Lange, H., Diekert, K., Kispal, G., and Lill, R. (2000) J. Biol. Chem. 275, 15955-61.

22. Morimoto, K., Nishio, K., and Nakai, M. (2002) FEBS Lett. 519 , $123-7$.

23. Wu, G., Mansy, S. S., Hemann, C., Hille, R., Surerus, K. K., and Cowan, J. A. (2002) J. Biol. Inorg. Chem. 7, 526-32.

24. Otwinowski, Z., and Minor, W. (1997) Methods Enzymol. 276, 307-26.

25. Brunger, A. T., Adams, P. D., Clore, G. M., DeLano, W. L., Gros, P., Grosse-Kunstleve, R. W., Jiang, J. S., Kuszewski, J., Nilges, M., Pannu, N. S., Read, R. J., Rice, L. M., Simonson, T., and Warren, G. L. (1998) Acta Crystallogr., Sect. D 54 (Pt 5), 90521

26. Collaborative Computational Project, Number 4 (1994) The CCP4 Suite: Programs for Protein Crystallography, Acta Crystallogr., Sect. D 50, 760-3.

27. Kleywegt, G. J., and Jones, T. A. (1996). Acta Crystallogr., Sect. D 52, 826-8.

28. Jones, T. A., Zou, J. Y., Cowan, S. W., and Kjeldgaard, M. (1991) Acta Crystallogr., Sect. A 47 (Pt 2), 110-9.

29. Murshudov, G. N., Vagin, A. A., and Dodson, E. J. (1997) Acta Crystallogr., Sect. D 53, 240-55.

30. Laskowski, R. A., MacArthur, M. W., Moss, D. S., and Thornton, J. M. (1993) J. Appl. Crystallogr. 26, 283-91.

31. Ollagnier-de Choudens, S., Nachin, L., Sanakis, Y., Loiseau, L., Barras, F., and Fontecave, M. (2003) J. Biol. Chem. 278, $17993-$ 8001.

32. Wu, S. P., and Cowan, J. A. (2003) Biochemistry 42, 5784-91.

33. Rees, D. C. (2002) Annu. Rev. Biochem. 71, 221-46.

34. Koradi, R., Billeter, M., and Wüthrich, K. (1996) J. Mol. Graphics $14,51-5$

35. POVRAY (http:/www.povray.org/).

36. Barton, G. J. (1993) Protein Eng. 6, 37-40.

BI035440S 